\title{
MARAN'S THEOREM (NEW THEOREM) ON RIGHT-ANGLED TRIANGLE
}

\author{
* A.K. Maran
}

\section{KEY WORDS}

Geometry, Triangle and its related theorems, Pythagoras theorems I and II, Appollonius theorem and Euclid's theorems I and II. American mathematical society subject classification index.

\section{Scope of Research}

In Geometric, right-angled triangle is one of the two-dimensional plane having three sides with one of its angle is $90^{\circ}$ and which is very important to solve problems related to Geometry and sometimes in other subject as well. Some fundamental concept /theorems of triangles are required to solve such problems and such theorems are (i) Pythagoras $1^{\text {st }}$ theorem ' (ii) Pythagoras $2^{\text {nd }}$ theorem (iii) Appollonius theorem (iv) Euclid's $1^{\text {st }}$ theorem ${ }^{2}$ and (v) Euclid's $2^{\text {nd }}$ theorem (Altitude theorem). ${ }^{3}$ In addition to these, the author has attempted to develop a new theorem related to right-angled triangle (Maran's theorem of right-angled triangle). The new theorem have been discussed and proved with relevant examples.

- Assistant Executive Engineer Nigilance) Ara. Kalai Maran, AEE(Vig), CSIR-H.Qrs, Anusondhan Bhavan, 2 Rafi Marg, New Delhi - 110001. 


\section{Maran's theorem on right-angled triangle}

If a right-angled triangle $A C B$ is divided into two parts viz., $A D C$ and $B D C$ by its altitude as its hypotenuse is its base (fig. 1) and these two triangles are superimposed over triangle $A B C$ as shown in figure. 2

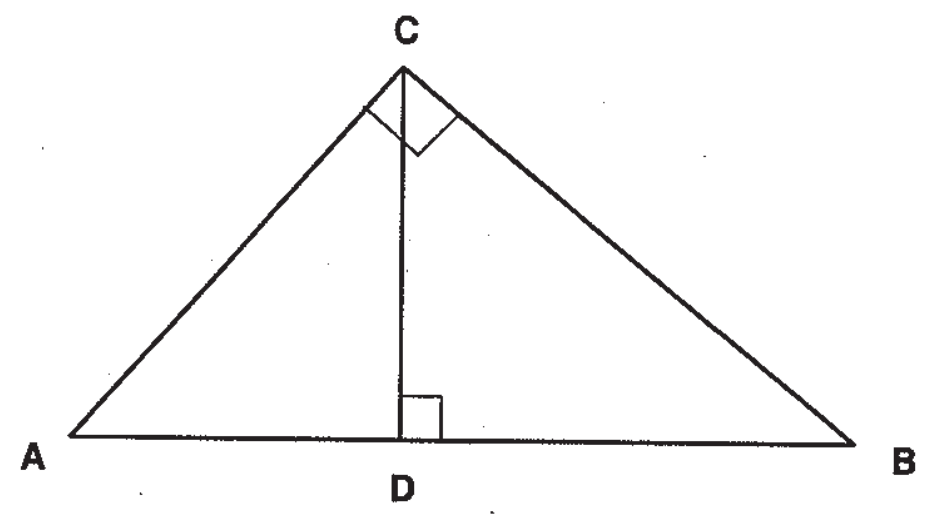

Fig.1: Typical example of Right angled Triangle ACB

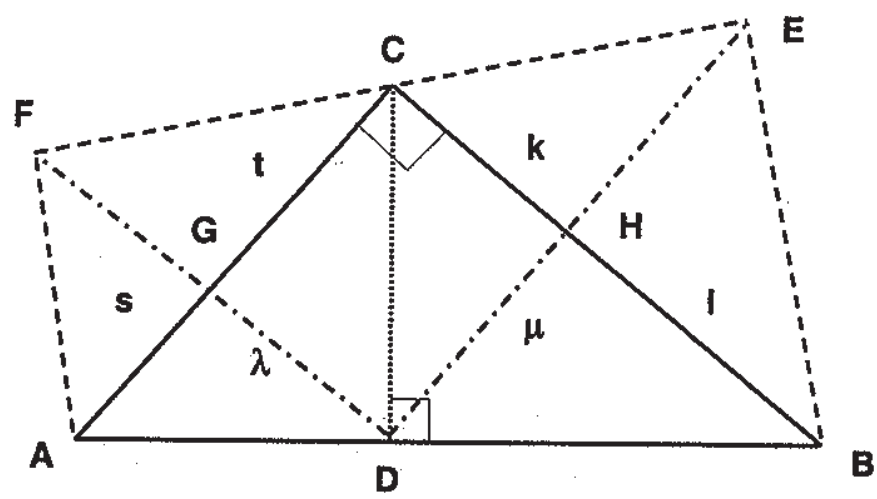

Fig.2: Superimposition of triangles over R.A.triangle ACB 
Let, $A C B$ is a right-angled triangle. $m \angle A C B=90^{\circ} . D$ is the point, which is projection of $C$ on line $A B$ i:e., line $C D \perp$ to $A B$. Let $C D$ is the altitude of the triangle, $D G=$ $\lambda, \mathrm{DH}=\mu, \mathrm{AG}=\mathrm{s}, \mathrm{CG}=t, \mathrm{CH}=\mathrm{k} \& \mathrm{BH}=1$. Therefore, $\mathrm{m} \angle \mathrm{ADC}=90^{\circ}$, $\mathrm{m} \angle \mathrm{BDC}=90^{\circ}, \triangle \mathrm{AFC} \equiv \triangle \mathrm{ADC}$ and $\triangle \mathrm{CEB} \equiv \triangle \mathrm{CDB}$.

Therefore, (i) The mathematical relation between $a, b, \lambda$ and $\mu$ is

$$
\lambda \times a=\mu \times b
$$

(ii) The mathematical relation between $k, l$, s and $t$ is

$$
k \times t=I \times s
$$

(iii) $\triangle E D F$ is similar to $\triangle A C B$

\section{Example}

See fig. 2. In this figure, a right-angled triangle $A C B, m \angle A C B=90^{\circ}, a=2 \sqrt{3}, b$ $=2, m=1, n=3, \lambda=\sqrt{3} / 2, \mu=3 / 2, k=\sqrt{3} / 2,1=3 \sqrt{3} / 2, s=1 / 2$ and $t=3 / 2$. Prove the above all theorems.

(i) The first theorem is that: $\lambda \times a=\mu \times b$ and substitute the values $a, b, \lambda \& \mu$ in LHS of the theorem,

$\lambda \times a=(\sqrt{3} / 2) \times(2 \sqrt{3})=3$

Substituting the values in RHS of the theorem,

$\mu \times b=(3 / 2) \times 2=3$

From $[1] \&[2], \lambda \times a=\mu \times b$ and hence the above first theorem has been proved.

(ii) The second theorem is that: $k \times t=s \times l$ and substitute the values $k, t, s \& l$ in LHS of the theorem,

$k \times t=(\sqrt{ } 3 / 2) \times(3 / 2)=(3 \sqrt{ } 3) / 4$

Substituting the values in RHS of the theorem,

$\mu \times b=(3 / 2) \times 2=3$

From [3] \& [4], $\mathrm{k} \times \mathrm{t}=\mathrm{s} \times 1$ and hence the second theorem has been proved. 
(iii) The third theorem is that: $\triangle E D F$ is similar to $\triangle A C B$

In triangle $A C B, \angle A C B=180^{\circ}-(\angle C A D+\angle C B D)=90^{\circ}$

Therefore, $A C B$ is a right-angled triangle

$\angle C A D=\operatorname{Cos}^{-1}(2 / 4)=\operatorname{Cos}^{-1}(1 / 2)=60^{\circ}$

$\angle C B D=\operatorname{Cos}^{-1}(2 \sqrt{3} / 4)=\operatorname{Cos}^{-1}(\sqrt{3} / 2)=30^{\circ}$

$\angle \mathrm{DFE}=\operatorname{Cos}^{-1}[(2 \sqrt{3} / 2) \div(2 \sqrt{3})]=\operatorname{Cos}^{-1}(1 / 2)=60^{\circ}$

$\angle D E F=\operatorname{Cos}^{-1}[(2 \times 3 / 2) \div(2 \sqrt{3})]=\operatorname{Cos}^{-1}(3 / 2 \sqrt{3})=30^{\circ}$

In triangle $\mathrm{FDE}, \angle \mathrm{FDE}=180^{\circ}-(\angle \mathrm{DFE}+\angle \mathrm{DEF})$

By substituting [7] \& [8] in above equation, we get $\angle \mathrm{FDE}=90^{\circ}$

From eqn $[5] \&[7], \angle \mathrm{CAD}=\angle \mathrm{DFE}$. Similarly, from eqn [6] \& [8], $\angle \mathrm{CBD}=\angle \mathrm{DEF}$

Therefore, $\triangle \mathrm{FDE}$ is a right-angled triangle. $\triangle \mathrm{FDE}$ is similar to $\triangle \mathrm{ACB}$ and hence the third theorem has also been proved.

\section{References}

1. W.Gellert.S.Gottwald, M.Hellwich. H.Kästner.H.Küstner (1989), The VNR Concise Encyclopedia of Mathematics, New york, Van Nostrand Reinhold, p.166.

2. W.Gellert.S.Gottwald, M.Hellwich. H.Kästner.H.Küstner(1989), The VNR Concise Encyclopedia of Mathematics, New york, Van Nostrand Reinhold, p.167.

3. W.Gellert.S.Gottwald, M.Hellwich. H.Köstner.H.Küstner(1989), The VNR Concise Encyclopedia of Mathematics, New york, Van Nostrand Reinhold, p.167. 